\title{
On The Nature Of The Higgs Boson
}

\author{
Damiano Anselmi \\ Dipartimento di Fisica "Enrico Fermi", Università di Pisa, \\ Largo B. Pontecorvo 3, 56127 Pisa, Italy \\ and INFN, Sezione di Pisa, \\ Largo B. Pontecorvo 3, 56127 Pisa, Italy \\ damiano.anselmi@unipi.it
}

\begin{abstract}
Several particles are not observed directly, but only through their decay products. We consider the possibility that they might be fakeons, i.e. fake particles, which mediate interactions but are not asymptotic states. A crucial role to determine the true nature of a particle is played by the imaginary parts of the one-loop radiative corrections, which are affected in nontrivial ways by the presence of fakeons in the loop. The knowledge we have today is sufficient to prove that most non directly observed particles are true physical particles. However, in the case of the Higgs boson the possibility that it might be a fakeon remains open. The issue can be resolved by means of precision measurements in existing and future accelerators.
\end{abstract}


In various respects, the Higgs boson has unique features. For example, it is a scalar field, unlike every other field of the standard model. Its key role is to trigger a crucial mechanism that gives masses to the particles. While it solves many problems, it leaves other questions unanswered. In this paper we study the possibility that it might hide a little secret. Specifically, the Higgs boson might be a "fake particle", i.e. an entity that resembles a true particle in various physical processes, but cannot be observed directly.

In quantum field theory, the poles of the free propagators are usually quantized by means of the Feynman prescription [1]. In that case, they describe physical particles. An alternative quantization prescription is able to quantize them as fake particles [2], or "fakeons" [3]. The fakeons are important in quantum gravity, because they allow us to build a consistent theory that is both unitary and renormalizable [2] (see also [4, 5]).

A fakeon simulates a physical particle when it mediates interactions or decays into physical particles. However, it is not an asymptotic state, because unitarity requires to project the fakeons away from the physical spectrum. In other words, a fakeon cannot be detected directly. An important physical prediction due to the fakeons is the violation of microcausality, which occurs at energies larger than their masses.

Quantum gravity predicts that at least one fakeon exists in nature [2]. It has spin 2, it is described by a symmetric tensor $\chi_{\mu \nu}$ and its mass $m_{\chi}$ could be much smaller than the Planck mass. Its free propagator has a negative residue at the pole, so $\chi_{\mu \nu}$ is a "fakeon minus" [6] and its dynamically generated width $\Gamma_{\chi}$ is negative.

The spin-2 gravifakeon is necessary to make the quantization of gravity consistent. In other sectors of high-energy physics, like the standard model in flat space, as well as its extensions, there might be no need of fake particles. However, if one fakeon exists in nature, it might not be the only one. Are there any other fakeons, maybe in the realm of the standard model? In this paper we provide enough arguments to exclude this possibility for most particles, but the cases of the Higgs boson and a few other particles remain unresolved.

Standard model extensions can be built by adding physical and fake particles, as long as they satisfy the conditions for the cancellation of the gauge anomalies and their masses are large enough to avoid conflict with the data. We do not explore these possibilities here, although they might have interesting applications. Instead, we inquire whether the particles that have already been identified so far are physical or fake.

Some particles, like the photon and the electron, are observed directly, so they are physical. Several other particles have not been observed directly, and probably will not 
be for a long time. We can mention the intermediate bosons, the Higgs field, the quarks, the gluons and the neutrinos. All of these are potentially fakeons. Since the definition of direct observation of a particle is to some extent debatable, we prefer to determine the true nature of all particles, including the photon and the electron, by means of indirect, more objective methods.

We show that, as of today, we have enough data to ensure that most particles are physical. However, we are unable to settle the matter in the cases of the Higgs boson, the top quark, the gluons and the right neutrinos. With the exception of the right neutrinos, the missing answers can be provided by precision measurements to be made in existing and/or future accelerators.

Before proceeding, let us recall a few properties. Physical and fake particles are quantized by means of different prescriptions, which are the Feynman prescription and the fakeon prescription. Introducing infinitesimal widths $\epsilon$ and $\mathcal{E}$, the unprescribed propagator $1 /\left(p^{2}-m^{2}\right)$ is turned into

$$
\frac{1}{p^{2}-m^{2}+i \epsilon}, \quad \frac{p^{2}-m^{2}}{\left(p^{2}-m^{2}\right)^{2}+\mathcal{E}^{4}},
$$

respectively. Note that, by convention, $\epsilon$ and $\mathcal{E}$ have different dimensions. The fakeon propagator vanishes on shell (which means for $p^{2}=m^{2}$ ) for every $\mathcal{E}>0$. This is a sign that is does not propagate a physical particle. Instead, the Feynman propagator blows up on shell. Off-shell, for $\left|p^{2}-m^{2}\right| \gg \epsilon, \mathcal{E}^{2}$, the two prescriptions are equivalent.

The sign in front of the Feynman propagator must be positive, otherwise it propagates a ghost, instead of a physical particle. Instead, the sign in front of the fakeon propagator can be either positive or negative, which distinguishes the "fakeon plus" from the "fakeon minus" [6]. The dynamically generated width $\Gamma$ of a fakeon plus (minus) is positive (negative).

In the right expression of (1) the poles of $1 /\left(p^{2}-m^{2}\right)$ are split into pairs of complex conjugates poles. Inside Feynman diagrams, the loop energy $p^{0}$ must be integrated along a path that passes under the left pair and over the right pair. The fakeon prescription needs to be specified by a number of other instructions, which we do not review here and have been recently summarized in ref. [6]. In the end, the most important property is that the fake particles can be consistently projected away from the physical spectrum.

At the tree level the difference between the two options (physical or fake) is just the projection, which has no impact on the particles that are not detected directly, like the vector bosons and the Higgs boson. The true nature of these particles can be established by 


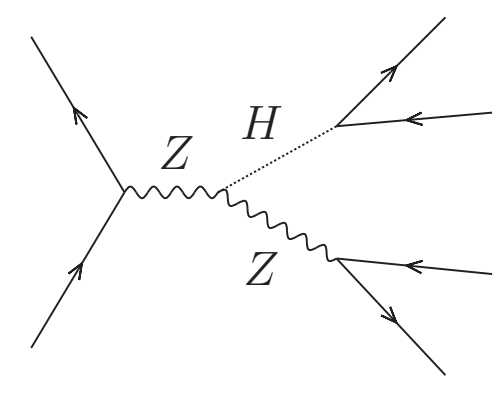

Figure 1: Higgs decay processes

analyzing the radiative corrections to the scattering processes. Since the physical spectrum is defined with respect to the broken phase, $S U(2)$ invariance is not very helpful. This means that the quantization prescriptions of the $W^{ \pm}$bosons, the $Z$ boson, the photon $\gamma$ and the Higgs field $\eta$ are in principle unrelated to one another.

To begin with, consider a process like the one shown in fig. 1 . Since $H$ and $Z$ decay, both quantization prescriptions (1) give the same prediction. This is a typical case where $H$ and $Z$ simulate real particles even if they are quantized as fakeons.

Specifically, it is possible to show $[5,6]$ that if we take a fakeon $F$ and resum the powers of its dynamical width $\Gamma_{F}$ into the so-called dressed propagator, the imaginary part of (minus) the $F$ dressed propagator tends to

$$
\pi Z_{F} \delta\left(p^{2}-m_{F}^{2}\right)
$$

in the limit $\left|\Gamma_{F}\right| \rightarrow 0$, as if the fakeon $F$ were indeed a physical particle of mass $m_{F}$, where $Z_{F}$ is the normalization factor. Applying these arguments to the final states of the process of fig. 1, we see no difference between true and fake $H$ and $Z$ particles.

Beyond the tree level, the two prescriptions give significantly different results for the imaginary parts $\mathcal{I}$ of the radiative corrections to the transition amplitudes [2, 3], while the real parts coincide in the two cases. At one loop, the relevant diagrams are self-energies, triangle diagrams and box diagrams. See fig. 2 for examples and refs. [7] for explicit formulas. Here we need to pay attention to the diagrams that contain at least one virtual fakeon and estimate the orders of magnitude of the various types of contributions. An important point is that the processes we are considering are far from the resonance peaks. In such conditions, the contributions of the three types of diagrams are of the same orders, so in many cases we can concentrate on the self-energies with no loss of generality. 
Consider a self-energy diagram $\mathcal{B}$ with internal legs of masses $m_{1}$ and $m_{2}$. The imaginary part $\mathcal{I}$ of $-i \mathcal{B}$ is equal to zero if an internal leg is quantized as a fakeon, while it is proportional to

$$
\theta\left(s-\left(m_{1}+m_{2}\right)^{2}\right) \sqrt{1-\frac{\left(m_{1}+m_{2}\right)^{2}}{s}} \sqrt{1-\frac{\left(m_{1}-m_{2}\right)^{2}}{s}}
$$

if both internal legs are quantized as physical particles, where $s$ is the center-of-mass energy squared. The real part of $-i \mathcal{B}$ is the same with both quantization prescriptions. Typically, when neither of the particles circulating in the loop are fakeons, $\mathcal{I}$ and the real part of $-i \mathcal{B}$ are of the same order, when $s$ is larger than the physical threshold $\left(m_{1}+m_{2}\right)^{2}$.

Let $\mathcal{I}_{c d}^{a b}$ denote the imaginary part of $-i$ times the bubble diagram that has $a, b$ as external legs and $c, d$ as circulating particles. If $a=b$, we just write $\mathcal{I}_{c d}^{a}$. Consider $\mathcal{I}_{W^{+} W^{-}}^{\gamma}, \mathcal{I}_{W^{+} W^{-}}^{Z}, \mathcal{I}_{W^{+} W^{-}}^{\gamma}$ and $\mathcal{I}_{Z H^{\prime}}^{Z}$. Since they have different thresholds, or depend on $s$ in different ways, it is possible to analyze their contributions separately in precision measurements. The imaginary parts $\mathcal{I}_{W^{+} W^{-}}^{\gamma}, \mathcal{I}_{W^{+} W^{-}}^{Z}$ and $\mathcal{I}_{W^{+} W^{-}}^{\gamma Z}$ contribute to the cross section $\sigma\left(e^{+} e^{-} \rightarrow\right.$ leptons, hadrons) and their thresholds $2 m_{W}$ are in the range of energies spanned for example by LEP II. Since no unexpected behavior has been noticed (and the data of LEP II are precise enough), we infer that $\mathcal{I}_{W^{+} W^{-}}^{a}$ are nonvanishing, hence the $W$ bosons are physical and not fake. The same can be said of the left neutrinos $\nu$, from $\mathcal{I}_{\nu \bar{\nu}}^{Z}$, the charged leptons $\ell$, from $\mathcal{I}_{\bar{\ell} \bar{\ell}}^{\gamma}, \mathcal{I}_{\ell \bar{\ell}}^{Z}, \mathcal{I}_{\ell \bar{\ell}}^{\gamma Z}$, and all the quarks $q$ but the top one, from $\mathcal{I}_{q \bar{q}}^{\gamma}$, $\mathcal{I}_{q \bar{q}}^{Z}, \mathcal{I}_{q \bar{q}}^{\gamma Z}$.

The case of the top quark $t$ remains unresolved. The self-energy contributions $\mathcal{I}_{t \bar{t}}^{\gamma}$, $\mathcal{I}_{t \bar{t}}^{Z}$ and $\mathcal{I}_{t \bar{t}}^{\gamma Z}$ with circulating tops have been missed by LEP II, due to their thresholds $2 m_{t} \sim 346 \mathrm{GeV}$. However, they can be studied in precision measurements with some effort of data analysis and background subtraction at LHC [8] and HiLumi [9], or, more directly, at the International Linear Collider [10], the Compact Linear Collider [11], the Future Circular Collider [12] and the Circular Electron Positron Collider [13], if they will be eventually built [14].

The analysis just made, based on the self-energies, leaves out the photon $\gamma$ and the $Z$ boson. We can prove that they are not fake by considering the box diagram of fig. 2 . Here the imaginary part associated with the vertical cut has a threshold equal to $m_{Z}$, so it contributes to the processes studied at LEP II. Since no discrepancies with respect to the predictions of the usual quantization prescription have been reported, we infer that both $\gamma$ and $Z$ are physical. As far as the $Z$ boson is concerned, we can also consider the box diagram with $\gamma$ replaced by a second $Z$, since the threshold $2 m_{Z}$ has also been exceeded 

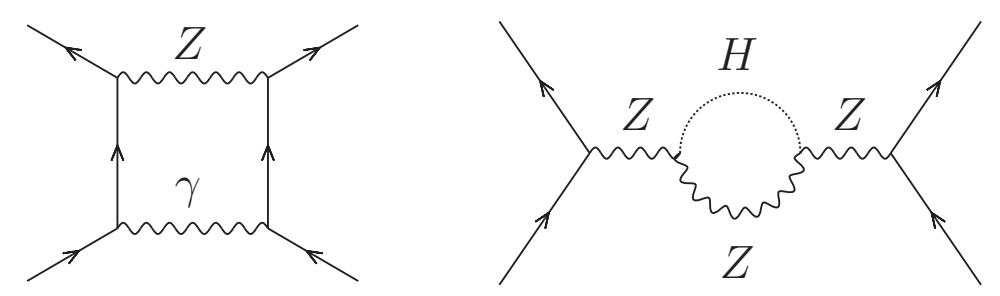

Figure 2: Relevant processes with $Z$ and $H$ bosons in loops

\section{by LEP II.}

Let us now consider the Higgs boson. If the Higgs field were a fakeon, it would have to be a fakeon plus, since the possibility that it might be a fakeon minus is excluded. Indeed, to turn it into a fakeon minus, we would have to flip the sign of its kinetic term $\left(D_{\mu} H^{\dagger}\right)\left(D^{\mu} H\right)$. Then the squared masses of $Z$ and $W^{ \pm}$would also turn into their opposites. However, it is not possible to quantize takyons as fakeons [both prescriptions (1) must have $\left.m^{2}>0\right]$, so we would have to flip the signs of the of $Z$ and $W^{ \pm}$kinetic terms as well. That would force us to quantize $Z$ and $W^{ \pm}$as fakeons (since the Feynman prescription would turn them into ghosts), which is contrary to the results obtained above. In the end, we remain with just two possibilities: the Higgs boson is a physical particle or a fakeon plus.

To decide which it is, consider the cross sections $\sigma\left(e^{+} e^{-} \rightarrow \gamma \ell j\right)$, where $\gamma \ell j$ denotes any final state made of photons, leptons and/or jets. If $H$ is physical, the imaginary part $\mathcal{I}_{Z H}^{Z}$ of the $Z$ self-energy with a Higgs field (see the right diagram of fig. 2) starts contributing from $\sqrt{s} \gtrsim m_{Z}+m_{H}=216 \mathrm{GeV}$. Enough above the threshold (say, at $\sqrt{s} \sim 240 \mathrm{GeV}$ ) the contribution of $\mathcal{I}_{Z H}^{Z}$ is comparable to the one of the real part. It is also comparable to the contributions of the imaginary parts of the other main $Z$ self-energy diagrams, like $\mathcal{I}_{W^{+} W^{-}}^{Z}$.

Thus, the difference between a physical Higgs boson and a fake one is important enough to be noticed, whenever a self-energy diagram like the one of fig. 2 contributes and the experiment is sensitive to it. If $\mathcal{I}_{Z H}^{Z}$ is found to be nonvanishing, then both $Z$ and $H$ are not fake. Instead, if $\mathcal{I}_{Z H}^{Z}$ is found to vanish, we conclude that $H$ must be fake, since we have already proved that $Z$ is not fake.

If LEP II had not stopped right below the threshold $m_{Z}+m_{H}$, we would already know the answer to this question. At present, the only possibility to fill this gap is to perform precision measurements at LHC or wait for HiLumi, ILC, CLIC, FCC or CEPC.

Other potentially relevant diagrams are the fermion self-energies that involve a virtual 


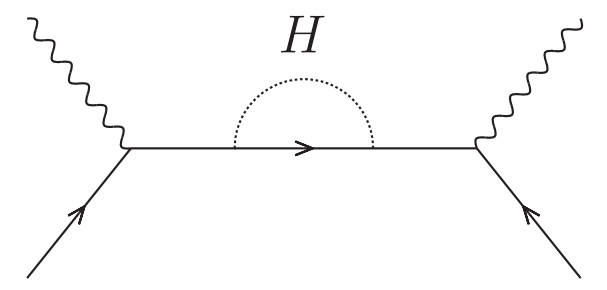

Figure 3: Compton-like process for fermion self-energy with Higgs boson

Higgs boson. The fermions $f$ must also be virtual, to turn on the imaginary part $\mathcal{I}_{f H}^{f}$, whose threshold is $m_{f}+m_{H}$. These self-energy diagrams contribute for example to the Compton-like process of fig. 3. However, the couplings of the fermions to the Higgs boson are suppressed by a factor $m_{f} / v$, where $v$ is the Higgs vev, and this ratio is squared in the diagram. The resulting contribution $\mathcal{I}_{f H}^{f}$ is too small to be observed in all cases apart from the one of the top quark, where the threshold raises to about $300 \mathrm{GeV}$. The virtual top quark can be produced by top-gluon and top-photon interactions, as well from the pairs $W^{+} b$ and $Z t$. These are processes that can be studied at LHC.

Replacing $H$ with a gluon in fig. 3, we obtain a contribution that allows us to test whether the gluons are physical or fake. In that case, it is enough to reach energies that are a bit larger than the quark mass and we can use any type of quark we want. We are not aware of data that can be immediately analyzed to obtain an answer in this case, but it is another problem that can be studied at LHC.

If we replace $Z$ or $\gamma$, or both, with $H$ in the box diagram of fig. 2 we obtain another interesting diagram with a virtual Higgs boson circulating in a loop. Then the fermions can only be top quarks, since, for the reasons recalled before, the couplings of $H$ to the other fermions are too small.

In conclusion, theoretical arguments and experimental evidence ensure that no particles of the standard model are fakeons, apart from possibly the Higgs boson and the top quark, the gluons and the right neutrinos. The top quark is related to other quarks by (approximate) family symmetries, which may suggest that it is probably physical. On the other hand, the possibility that the Higgs boson is a fakeon is more intriguing, given the peculiarities of this particle. All cases, apart from the one of the right neutrinos, can be settled in future experiments or by performing precision measurements at LHC. If one or more particles of the standard model turn out to be fakeons, it becomes interesting to devise specific experiments to search for the first signs of violations of microcausality. 


\section{Acknowledgments}

We are grateful to U. Aglietti, M. Grazzini, M. Piva and A. Strumia for useful discussions.

\section{References}

[1] See for example, M.E. Peskin and D.V. Schroeder, An introduction to quantum field theory, Westview Press, 1995, § 4.4.

[2] D. Anselmi, On the quantum field theory of the gravitational interactions, J. High Energy Phys. 06 (2017) 086, 17A3 Renormalization.com and arXiv:1704.07728 [hep-th].

[3] D. Anselmi, Fakeons and Lee-Wick models, J. High Energy Phys. 02 (2018) 141, 18A1 Renormalization.com and arXiv:1801.00915 [hep-th].

[4] D. Anselmi and M. Piva, The ultraviolet behavior of quantum gravity, J. High Energ. Phys. 05 (2018) 27, 18A2 Renormalization.com and arXiv:1803.07777 [hep-th].

[5] D. Anselmi and M. Piva, Quantum gravity, fakeons and microcausality, J. High Energy Phys. in press, 18A3 Renormalization.com and arXiv:1806.03605 [hep-th].

[6] D. Anselmi, Fakeons, microcausality and the classical limit of quantum gravity, 18A4 Renormalization.com and arXiv:1809.05037 [hep-th].

[7] W.L. van Neerven, Dimensional regularization of mass and infrared singularities in two loop on-shell vertex functions, Nucl. Phys. B268 (1986) 453;

M. Bohm, W. Hollik and H. Spiesberger, On the 1-loop renormalization of the electroweak standard model and its applications to leptonic processes, Fortschr. Phys. 34 (1986) 11;

M. Consoli and W. Hollik, Electroweak radiative corrections for Z physics, in Z physics at LEP 1, Vol. 1: Standard physics, G. Altarelli, R. Kleiss and C. Verzegnassi Editors, CERN 89-08. 
[8] For updates on recent precision measurements, see The ATLAS collaboration, $Z Z \rightarrow \ell^{+} \ell^{-} \ell^{\prime+} \ell^{\prime-}$ cross-section measurements and search for anomalous triple gauge couplings in $13 \mathrm{TeV}$ pp collisions with the ATLAS detector, Phys. Rev. D 97 (2018) 032005 and arXiv:1709.07703 [hep-ex];

The ATLAS collaboration, Precision measurement and interpretation of inclusive $W^{+}, W^{-}$and $Z / \gamma^{*}$ production cross sections with the ATLAS detector, Eur. Phys. J. C 77 (2017) 367 and arXiv:1612.03016 [hep-ex].

[9] High Luminosity LHC, website: http://hilumilhc.web.cern.ch/

[10] International Linear Collider, website: http://www.linearcollider.org/ILC

[11] Compact Linear Collider, website: http://clic-study.web.cern.ch/

[12] Future Circular Collider, website: https://fcc.web.cern.ch/

[13] Circular Electron Positron Collider, website: http://cepc.ihep.ac.cn/

[14] For a recent assessment on future perspectives see, for example, M. Mangano, LHC and beyond: future perspective for High Energy Physics, New Frontiers in Theoretical Physics, XXXVI Convegno Nazionale di Fisica Teorica, May 2018, Cortona, Italy. 